Document downloaded from:

http://hdl.handle.net/10251/81218

This paper must be cited as:

Pérez-Aparicio, JL.; Palma, R.; Taylor, R. (2012). Finite element analysis and material sensitivity of Peltier thermoelectric cells coolers. International Journal of Heat and Mass Transfer. 55(4):1363-1374. doi:10.1016/j.ijheatmasstransfer.2011.08.031.

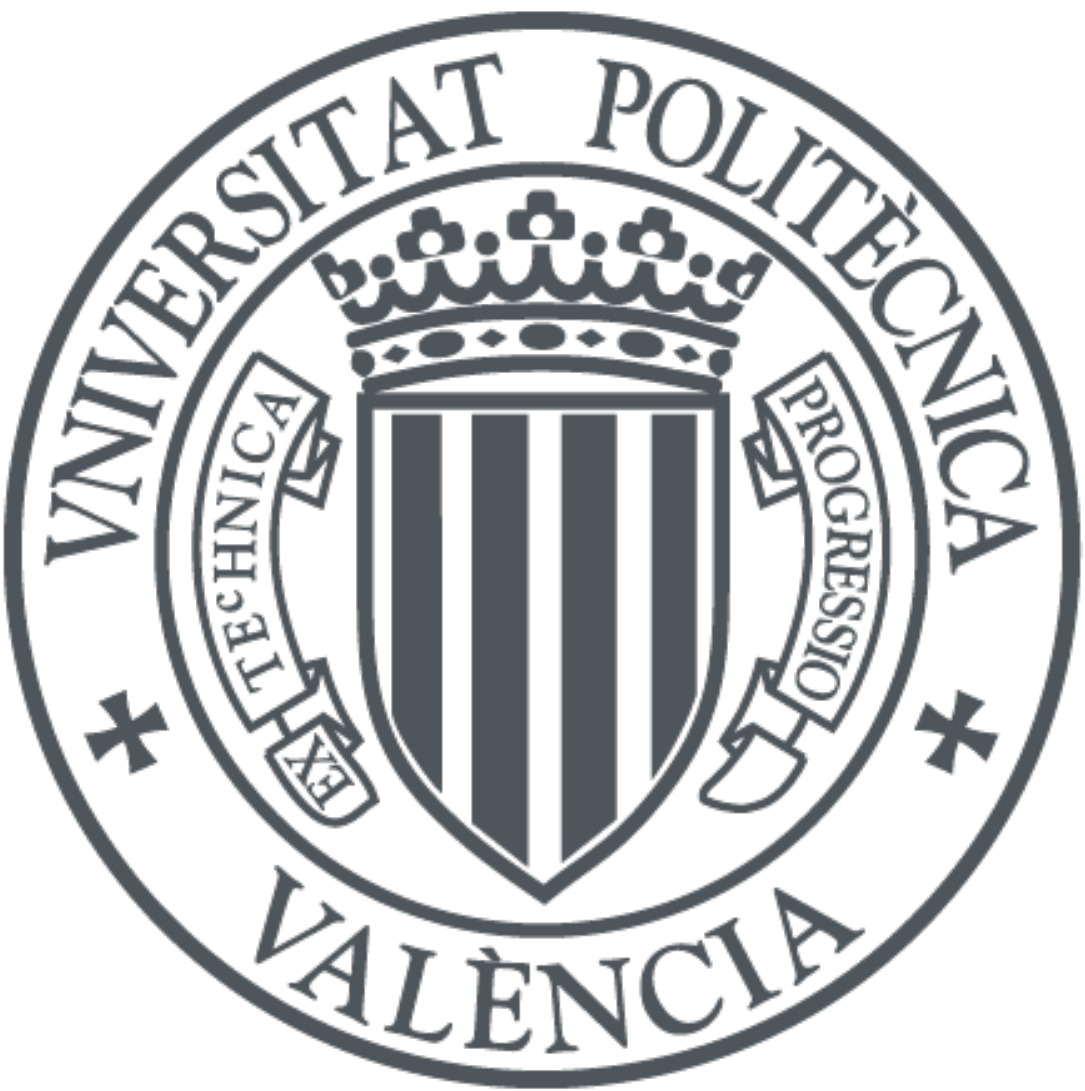

The final publication is available at

http://dx.doi.org/10.1016/j.ijheatmasstransfer.2011.08.031

Copyright Elsevier

Additional Information 


\title{
Finite Element Analysis and Material Sensitivity of Peltier Thermoelectric Cells Coolers
}

\author{
J.L. Pérez-Aparicio ${ }^{\mathrm{a}}$, R. Palma ${ }^{\mathrm{a}}$, R.L. Taylor ${ }^{\mathrm{b}}$ \\ ${ }^{a}$ Mecánica de Medios Continuos y Teoría de Estructuras, Universidad Politécnica de Valencia, Spain \\ ${ }^{b}$ Department of Civil and Environmental Engineering, University of California at Berkeley, California, USA
}

\begin{abstract}
In this work, a finite element simulation of a commercial thermoelectric cell, working as a cooling heat pump, is presented. The finite element is three-dimensional, non-linear in its formulation (using quadratic temperature-dependence on material properties) and fully coupled, including the Seebeck, Peltier, Thomson and Joule effects. Another special interface finite element is developed to prescribe the electric intensity, taking advantage of repetitions and symmetries. A thorough study of the distributions of voltage, temperature and the corresponding fluxes is presented, and the performance of the cell is compared with those of the manufacturer and simplified analytical formulations, showing a good agreement with the former. Combining the finite element model with the Monte Carlo technique, a Sensitivity Analysis is presented to take into account the performance variables dependence on the material properties, geometrical parameters and prescribed values. This analysis, which can be considered a first step to optimize these devices, concludes that the temperature-dependence of the material properties such as electric conductivity and Seebeck coefficient is very relevant on the cell performance.
\end{abstract}

Keywords:

Thermoelectric coolers, Non-linear FEM, Monolithic full coupling, Seebeck, Peltier, Thomson, Joule, Sensitivity Analysis, Monte Carlo.

\section{Introduction}

Peltier Thermoelectric Cells (TEC) are devices composed of several Thermocouples (TC), thermally connected in parallel and electrically in series. TC's are formed by pairs of n- and $\mathrm{p}-$ type Thermoelements (TE), which are solid state semiconductors denominated Thermoelectric Materials (TM). TM's convert temperature gradients to electric voltages and vice versa, by means of three (Seebeck, Peltier and Thomson) separated effects. The TEC can be used in two ways: heat pumps (working in cooling or heating modes) and electric generators. The present work studies the cooling TEC, that is energetically not efficient but has several advantages such as compactness, simplicity and reliability (lack of moving parts). For this reason, cooling TEC's are applied to stabilize the temperature of laser diodes, to cool infrared detectors and to small-scale refrigeration, see [1] for a full revision on applications.

The coefficient-of-performance (COP) is defined as the cooling capacity divided by the consumed electric power, and depends on the geometrical and material properties of the TEC. Many works study the COP analytically and numerically; from an analytical point of view, in [2] there is an expression of the COP using two main simplifications: the Thomson effect is not considered and any material properties are function of the temperature. The first simplification was addressed in [3], where it was analytically (with rather simple formulae) concluded that the influence of Thomson on COP is about $2 \%$. This influence was further explained in [4], reporting qualitatively that the fraction of both Fourier and Joule heating at the cold face is reduced by Thomson. The second simplification was studied in [5], concluding that it is very important to consider temperature-dependent properties to design high-performance TEC's. From a numerical point of view, [6], [7] and [8] developed a steady-state and nonlinear (including constitutive and Joule heating) 3-D Finite Element (FE) to study the thermal, voltage and flux distributions inside a TE. Recently, [9] and [10] implemented thermoelectric elements into the Finite Element Method (FEM) commercial software. These works were validated by analytical and experimental results, respectively. Furthermore, the FE used by [9] included a standard interface element to model heat convection. The COP is reduced when the convection and radiation are elevated, according to the 3-D finite difference model developed by [11]. The previous works considered deterministic material and geometrical properties, but not their inherent randomness. In this sense, in [12] it was reported the treatment of physical uncertainties is a research area of great importance for the continuum mechanics community.

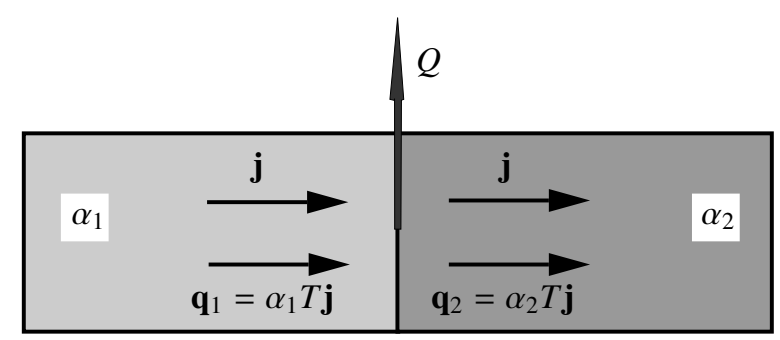

Figure 1: Peltier effect fluxes in two thermoelectric materials. 
In the current work, a specially developed interface element that takes into account convection and radiation is incorporated to the completely non-linear FE described in [8]. These two FE are used to simulate a commercial TEC fabricated by Melcor [13], comparing FEM and manufacturer results. Furthermore, temperature, voltage and flux distributions within the TEC are obtained and discussed. Finally, the combined Monte CarloFEM technique developed in [14] is applied to obtain uncertainties and sensitivities for the COP, relative to design variables that can be important. This probabilistic study permits the selection of the most relevant design variables, which could be taken into account to fabricate TEC's and to optimize its performance.

\section{Basic Phenomena}

In this section, an overview of the basic phenomena that influences the functioning of Peltier TC's is presented. For a detailed description, most of the related formulation has been published in [8].

\subsection{The Peltier and Thomson effects}

In a TM, if the temperature $T$ (always in absolute Kelvin degrees) is not constant, an electromotive force $\varepsilon=-\alpha \nabla T$ appears ( $\alpha$ is the absolute Seebeck coefficient). This force depends on the material characteristics and on the temperature itself, and is equal to an electric potential gradient superimposed to that from Ohm's Law:

$$
\mathbf{j}=-\gamma \nabla V-\alpha \gamma \nabla T
$$

where $\gamma$ is the electric conductivity. This effect has been traditionally used to measure temperatures with basic thermocouples. For the heat transfer field, a similar description holds: the charges (either electrons or holes) from the electric current can transport through the material thermal energy in a sort of convection phenomena that is superimposed to that of Fourier's Law.

In a TM, $\alpha$ can also be defined as the entropy per electric charge unit; therefore, when $T$ is constant the electric flux $\mathbf{j}$ transports an entropy flux per unit surface and unit time $\mathbf{j}_{s}=\alpha \mathbf{j}$. Along with this entropy transport, there will be a heat transfer $\mathbf{q}=T \mathbf{j}_{s}$. Superimposing this flux to that created by the temperature gradient, and using (1):

$$
\mathbf{q}=-\kappa \nabla T+\alpha T \mathbf{j}=-\alpha \gamma T \nabla V-\left(\kappa+\alpha^{2} \gamma T\right) \nabla T
$$

where $\kappa$ is the thermal conductivity.

In Figure 1, an electric flux $\mathbf{j}$ is represented passing through the union of two thermoelectric parts with different $\alpha$. This flux is taken constant through the interface of area $A_{i}$ by the hypothesis of conservation of charge. Ignoring for now the thermal conduction influence and assuming constant the temperature at the interface, the coupled heat flux will be $\mathbf{q}=\alpha T \mathbf{j}$. Since the two materials different $\alpha$ 's, a jump of $\mathbf{q}$ will appear at the interface, inducing the presence of a heat power $Q=\left(\mathbf{q}_{1}-\mathbf{q}_{2}\right) \cdot \mathbf{n} A_{i}=\left(\alpha_{1}-\alpha_{2}\right) T \mathbf{j} \cdot \mathbf{n} A_{i}$, where $\pi_{12}=\alpha_{1}-\alpha_{2}$ is the absolute Peltier coefficient. If $\alpha_{1}>\alpha_{2}$ there will be a release of heat, otherwise an absorption. This phenomenon is called Peltier, and in reality is more complicated than the one described here, due to the temperature high non-linearity at the interphase (see [8]); $T$ reduces/increases at the center of this interphase in order to "absorb" also by conduction the Peltier heat, creating a strong gradient.

From $\mathbf{q}=\alpha T \mathbf{j}$, it can be appreciated that this heat flux will change from one point to other if $\alpha$ also changes, in particular if it is a function of the varying temperature field (see Figure 4). This is the Thomson effect, not always negligible in TM due to possible high temperature gradients and variations of $\alpha$ with this temperature.

Equations (1) and (2) are called constitutive and compatibility equations in Computational Mechanics and are used for the formulation and implementation of a special finite element.

\subsection{Peltier Cells}

Peltier thermocells, or as before TEC are usually composed of many special TC of the type from Figure 2. These TC are composed of two parallelepiped TM, each called pellet or TE, with different $\alpha$ coefficients and able to refrigerate without moving parts. They are connected electrically in series by copper bars and tin solders, to form a circuit fed by a source $V_{a f}$ with an intensity $I_{t e c}$. Thermally, they are connected in parallel with external plate-shaped alumina $\mathrm{Al}_{2} \mathrm{O}_{3}$ with a reasonable thermal conductivity, that is used to isolate electrically the TC.

In the cooling mode studied in this work, the TEC takes heat from the cold face at $T_{c}$ and transports it to the hot face at $T_{h}$. In order to maximize this transport, $\pi_{n p}$ also needs to be maximized. Therefore, the Seebeck coefficients must be as different as possible, for instance from semiconductors doped positively $\alpha_{p}>0$ and negatively $\alpha_{n}<0, \alpha_{n}=-\alpha_{p}$. The intensity $I_{t e c}$ circulates upwards in the TE with property $\alpha_{n}$ and downwards in the other, therefore due to the different signs the Peltier heat flux $\mathbf{q}$ is directed down in both. The same device can act in heating mode if the electric current is driven from the $\mathrm{p}-$ to the n-type TE.

Two main detrimental effects (irreversibilities) have to be taken into consideration: i) heat conduction from the hot face to the cold face, and ii) internal heat generated by the Joule effect. For the latter, in analytical formulae it is assumed (sometimes with little accuracy) that half the heat goes to the hot face and the other half to the cold face. From [2], the approximate expressions for the total heat taken from the cold face $Q_{c}$ and that given to the hot face $Q_{h}$ are

$$
\left\{\begin{array}{l}
Q_{h}=\left(\alpha_{p}-\alpha_{n}\right) I_{t e c} T_{h}-\frac{\kappa A}{L}\left(T_{h}-T_{c}\right)+\frac{L}{2 \gamma A} I_{t e c}^{2} \\
Q_{c}=\left(\alpha_{p}-\alpha_{n}\right) I_{t e c} T_{c}-\frac{\kappa A}{L}\left(T_{h}-T_{c}\right)-\frac{L}{2 \gamma A} I_{t e c}^{2}
\end{array}\right.
$$

where the three terms represent the Peltier, Fourier and Joule effects, and $A, L$ are the areas and lengths of the TE. In this simplified expression Thomson is not included; however, [3] and [5] reported analytical expressions that take it into account. These works concluded that the influence on the analytical $Q_{c}$, 


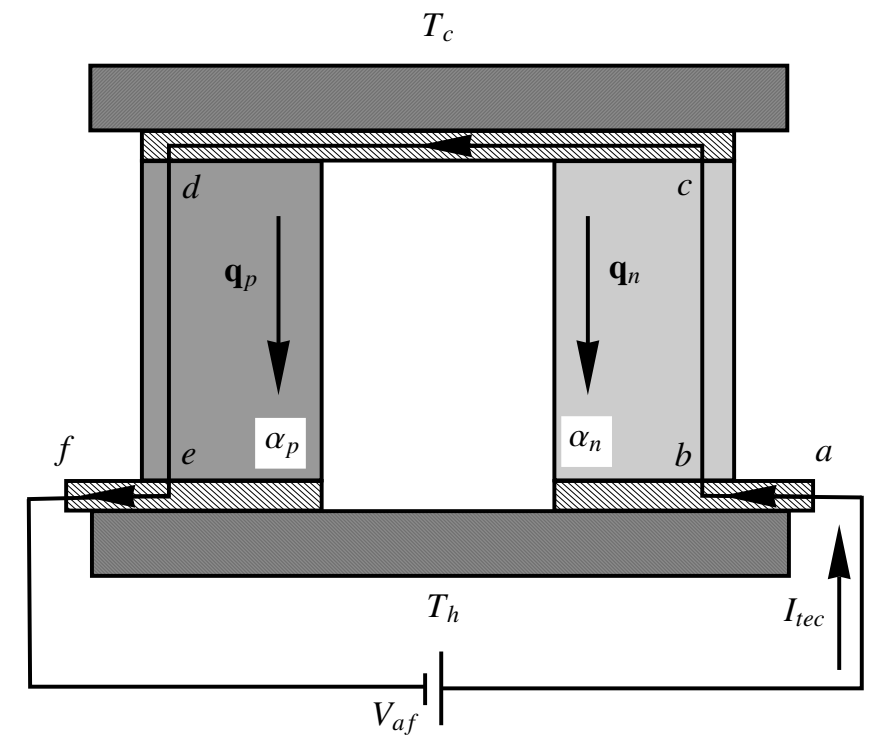

Figure 2: Peltier cooling thermocouple and fluxes.

$Q_{h}$ (not necessarily on the experimental ones) of Thomson is of only about $2 \%$.

If perfect performance is assumed, the difference between this two heats must be equal to the electric power, as in (4) top. The maximum intensity that can pass the TEC is calculated from the minimum of $Q_{c}$ with respect to $I_{t e c}$, giving the middle equation. Finally, in the bottom the maximum increment of the temperature $\Delta T_{\max }=T_{h}-T_{c}$ is obtained from $Q_{c}\left(I_{\max }\right)=0$ :

$$
\begin{aligned}
V_{a f} I_{\text {tec }} & =\left(\alpha_{p}-\alpha_{n}\right) I_{\text {tec }}\left(T_{h}-T_{c}\right)+\frac{L}{\gamma A} I_{\text {tec }}^{2} \\
I_{\text {max }} & =\frac{\kappa A}{L\left(\alpha_{p}-\alpha_{n}\right)}\left(\sqrt{1+2 T_{h} Z}-1\right) \\
\Delta T_{\text {max }} & =T_{h}-\frac{\sqrt{1+2 T_{h} Z}-1}{Z}
\end{aligned}
$$

where the material figure of merit is $Z=\alpha^{2} \gamma / \kappa$. Notice that $V_{a f}$ has to be greater than the voltage gradient created by the Peltier effect, related to the temperature gradient.

Finally, the coefficient-of-performance COP is defined as the total heat extracted from the cold face divided by the consumed electric power:

$$
\mathrm{COP}=\frac{Q_{c}}{V_{a f} I_{t e c}}
$$

\subsection{Carnot equivalence}

In a first analysis, it could seem that the Peltier cooling violates the thermodynamic laws, but in reality it follows the Carnot cycle of refrigeration, neglecting the two mentioned irreversibilities for now. To understand this cycle, let us study the path of a charge carrier from point $c$ to $d$ in Figure 3. In $c, d$ the carrier is approximately at temperature $T_{c}$, while in $b, e$ is at a higher $T_{h}$. On the other hand, in $b, c$ the entropy $\left(\alpha_{n}\right)$ is lower than that of $d, e$. This situation can be represented in the
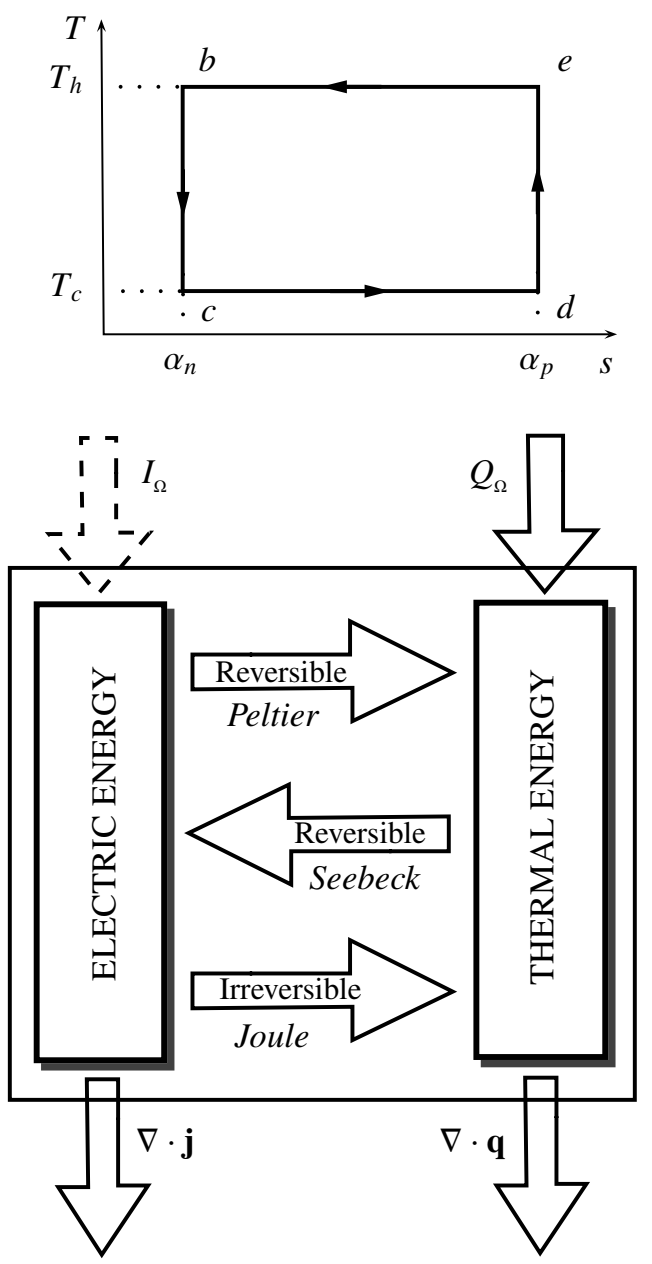

Figure 3: Simplified $T-s$ diagram of a charge carrier in thermoelectric materials (top). Balance of energies per unit volume (bottom). The non-physical term $I_{\Omega}$ is included for numerical procedures.

classical simplified $T-s$ Carnot cycle of Figure 3 top, in which the following processes can be distinguish:

- Isentropic expansion $b-c$ along the n-type TE. Not an expansion as in gases but of similar effect: the charge carrier performs a work (electric energy generated by Seebeck) under a constant entropy

- Isothermal heat absorption $c-d$ along the cold face. The carrier absorbs heat (Peltier) from the cold face at constant temperature incrementing its entropy

- Isentropic compression $d-e$ along the p-type TE. The entropy remains constant but the carrier absorbs electric work

- Isothermal heat transfer $e-b$ along the hot face. At constant $T_{h}$, the carrier releases heat decreasing its entropy

The expansion and compression would be not isentropic (a heat exchanged would exist) if Thomson is considered. See [15], that includes it, and [16] that includes Thomson, Fourier and Joule. 


\begin{tabular}{|l|c|c|c|}
\hline & $\alpha \times 10^{-4}$ & $\gamma \times 10^{5}$ & $\gamma \times 10^{5}$ \\
\hline \hline Units & {$[\mathrm{V} / \mathrm{K}]$} & {$[\mathrm{A} /(\mathrm{V} \cdot \mathrm{m})]$} & {$[\mathrm{A} /(\mathrm{V} \cdot \mathrm{m})]$} \\
\hline $\mathrm{Al}_{2} \mathrm{O}_{3}$ & 0 & 0 & 35.3 \\
\hline $\mathrm{Bi}_{2} \mathrm{Te}_{3} \mathrm{n}$ & -2.042 & 1.018 & 1.616 \\
\hline $\mathrm{Bi}_{2} \mathrm{Te} e_{3} \mathrm{p}$ & 2.042 & 1.018 & 1.616 \\
\hline $\mathrm{Cu}$ & 0 & 581 & 386 \\
\hline $\mathrm{Sn} \mathrm{Pb}$ & 0 & 47 & 48 \\
\hline
\end{tabular}

Table 1: Material properties at $15^{\circ} \mathrm{C}$.

\subsection{Material Properties}

The TM properties are taken from [2] and depicted in Figure 4 for $\mathrm{Bi}_{2} \mathrm{Te}_{3}$, a metal-metalloid alloy showing a high $\alpha$ although with a strong dependency on $T$, as well as $\kappa$ and $\gamma$. The TM properties can be fitted to the quadratic polynomial:

$$
\begin{aligned}
& \alpha=\underbrace{1.988 \times 10^{-4}}_{\alpha_{0}}+\underbrace{3.353 \times 10^{-7}}_{\alpha_{1}} T-\underbrace{7.521 \times \times^{-10}}_{\alpha_{2}} T^{2} \\
& \kappa=\underbrace{1.663}_{\kappa_{0}}-\underbrace{3.580 \times 10^{-3}}_{\kappa_{1}} T+\underbrace{3.195 \times \times^{-5}}_{\kappa_{2}} T^{2} \\
& \gamma=\underbrace{1.096 \times 10^{5}}_{\gamma_{0}}-\underbrace{5.590 \times 10^{2}}_{\gamma_{1}} T+\underbrace{2.498}_{\gamma_{2}} T^{2}
\end{aligned}
$$

where the temperature is introduced in Celsius degrees. The other materials properties, assumed constant with $T$, are obtained from [13] and listed in Table 1.

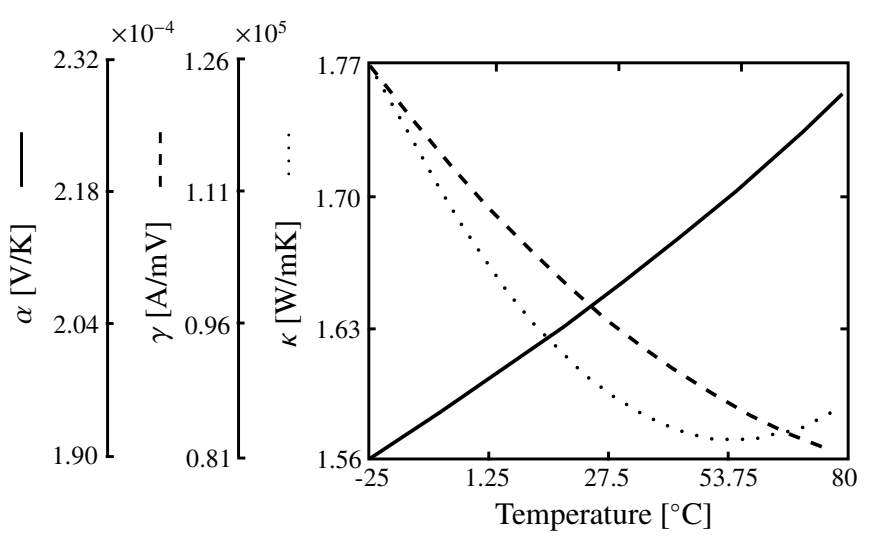

Figure 4: Thermoelectric material property variations with temperature.

\section{Equilibrium equations}

In Figure 3 bottom, the balance of energies per unit volume that occur inside an isolated TM is schematized. The external arrows at the bottom represent the energy interchange with the exterior, the external ones at the top the energy generated inside the volume and finally the internal ones the transformation from thermal to electric or vice versa. Note that Thomson is not included in the balance of energies and the intensity $I_{\Omega}$ does not have physical meaning since from charge conservation must be zero, but is kept in this article since can be useful to introduce prescribed electric fluxes in a volumetric form.

The static equilibrium equations are derived from the balances of electric charge and of energy. The first one expresses the equality of the quantity of electric flux $\mathbf{j}$ flowing through the boundary $\Gamma$ and the electric charge $I_{\Omega}$ created inside the volume $\Omega$. The local form of the balance equation is obtained using the divergence theorem:

$$
-\oint_{\Gamma} \mathbf{j} \cdot \mathbf{n} \mathrm{d} \Gamma+\int_{\Omega} I_{\Omega} \mathrm{d} \Omega=
$$

$$
-\int_{\Omega} \nabla \cdot \mathbf{j} \mathrm{d} \Omega+\int_{\Omega} I_{\Omega} \mathrm{d} \Omega=0 \quad \Rightarrow \quad \nabla \cdot \mathbf{j}=I_{\Omega}
$$

where the electric flux that exits the boundary is considered negative by convention.

The second balance equation accounts for three energy interchanges. First, the thermal energy $Q_{\Omega}$ created inside the volume per unit time, second the thermal energy that crosses the boundary obtained using the divergence theorem and last the electric energy that crosses the boundary, obtained from (7) and again the divergence theorem

$$
\begin{aligned}
& \mathcal{E}_{1}=\int_{\Omega} Q_{\Omega} \mathrm{d} \Omega \\
& \mathcal{E}_{2}=-\oint_{\Gamma} \mathbf{q} \cdot \mathbf{n} \mathrm{d} \Gamma=-\int_{\Omega} \nabla \cdot \mathbf{q} \mathrm{d} \Omega \\
& \mathcal{E}_{3}=-\oint_{\Gamma}(V \mathbf{j}) \cdot \mathbf{n} \mathrm{d} \Gamma=-\int_{\Omega} \mathbf{j} \cdot \nabla V \mathrm{~d} \Omega-\int_{\Omega} V I_{\Omega} \mathrm{d} \Omega
\end{aligned}
$$

Again for an arbitrary volume $\Omega$, equilibrium of the three energies $\mathcal{E}_{1}+\mathcal{E}_{2}+\mathcal{E}_{3}=0$ gives the second equilibrium equation (see [8], for details)

$$
\nabla \cdot \mathbf{q}+\mathbf{j} \cdot \nabla V=Q_{\Omega}-V I_{\Omega}
$$

The electric boundary conditions are the prescribed voltage $V=V_{c}$ on $\Gamma_{V}$ and the electric flux $\mathbf{j} \cdot \mathbf{n}=j_{c}$ on $\Gamma_{j}$. Correspondingly, $T=T_{c}$ on $\Gamma_{T}$ and $\mathbf{q} \cdot \mathbf{n}=q_{c}$ on $\Gamma_{q}$ for the thermal field. Notice that $\Gamma_{V}$ and $\Gamma_{j}$ on one hand, and $\Gamma_{T}$ and $\Gamma_{q}$ on the other do not overlap, but that electric and thermal boundaries are completely independent.

\section{Finite Element development}

Equations (1), (2), (7) and (8) along with proper boundary conditions can be transformed into a weak (integral) form and from that into a matrix form amenable to be solved by a computer. The choice of the interpolation functions is what defines the FEM.

In this work, we are interested in the global performance of TEC but also in the details of voltage, temperature, electric and heat fluxes that can affect the accuracy of the approximated expressions (3), (4) and (5). Therefore, a three-dimensional isoparametric element was implemented in the research code FEAP [17] in order to capture any interesting phenomenon. The 
element technology is simple in the FE sense, but the governing equations are strongly non-linear for two reasons: i) the material dependency on $T$, and ii) the Joule effect. The former influences all formulation terms, and in some temperature ranges (e.g. around $54^{\circ} \mathrm{C}$, see Figure 4), it is clearly non admissible to use constant or even linear approximations. The details of the finite element derivation are given in [8] and will not be repeated here. The left Figure 5 depicts a scheme of this element, in which basic Lagrangian shape functions are used. Voltage and temperature are the nodal degrees of freedom, and fluxes are obtained by derivation.

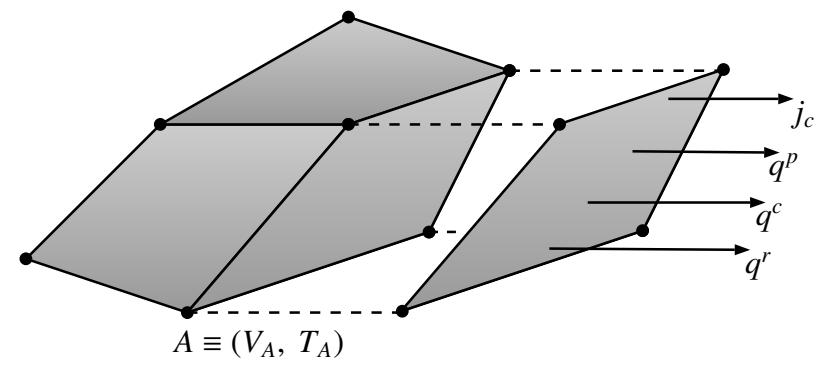

Figure 5: Non-linear isoparametric 3-D thermoelectric finite element (left). Radiation and convection 2-D interface finite element (right).

In TEC, usually the TC are arranged very closely, to allow the vertical heat transfer to be uniform. Due to the trend towards miniaturization in electronic and other devices, this tendency has recently been reinforced. Temperatures can also reach very high values not usual in traditional mesoscale applications.

Therefore, the interchange of heat flux between TE of the same or different TC can be important; an special interface 2-D element has been developed in this article to simulate radiation and convection heat fluxes through the air, avoiding the expensive FE meshing of this gas. To facilitate the assembly process, this new element has also voltage and temperature as degrees of freedom in each node, which are interpolated using standard shape functions, see [18]:

$$
V \approx V^{h}=N_{A} V_{A} ; \quad T \approx T^{h}=N_{A} T_{A}
$$

Furthermore, isoparametric concepts are considered and the spatial coordinates approximated by $\mathbf{x}=N_{A}(\boldsymbol{\xi}) \mathbf{x}_{A}$, where $\boldsymbol{\xi}$ are the natural coordinates and $A$, and later $B$ the global node numbers.

The interface element does not represent any physical continuum, therefore it is uncoupled but non-linear since the radiation phenomena depends on the function $T^{4}$. From a FEM point of view, the Newton-Raphson scheme has to be used to solve this non-linearity and the governing equations are written in residual form:

$$
R_{V_{A}}=-\int_{\Gamma_{j}} N_{A} j_{c} \mathrm{~d} \Gamma ; \quad R_{T_{A}}=-\int_{\Gamma_{q}} N_{A} q_{c} \mathrm{~d} \Gamma
$$

where $q_{c}$ now is composed of the prescribed $q^{p}$, the convection $q^{c}$ and the radiation $q^{r}$ heat fluxes:

$$
q_{c}=q^{p}+\underbrace{h\left(T-T_{\infty}\right)}_{q^{c}}+\underbrace{\epsilon \sigma\left(T^{4}-T_{\infty}^{4}\right)}_{q^{r}}
$$

and where $h$ is the convection heat transfer coefficient that depends on the physical properties of the surrounding fluid (such as temperature and speed) and the physical situation in which convection occurs, and $\epsilon$ the emissivity. According to the data given by the manufacturer and those used in [9] and [4], in the present work it is assumed $h=10\left[\mathrm{~W} / \mathrm{m}^{2} \mathrm{~K}\right]$ and $\epsilon=0.02$ [-]. The $\sigma=5.67 \times 10^{-8}\left[\mathrm{~W} /\left(\mathrm{m}^{2} \cdot \mathrm{K}^{4}\right)\right]$ is the universal StefanBoltzmann constant and $T_{\infty}=300[\mathrm{~K}]$ the reference temperature.

The consistent tangent matrix is divided into four submatrices, corresponding to the negative derivatives of (10) with respect to the discretization of the two degrees of freedom:

$$
\begin{aligned}
& K_{V V_{A B}}=0 ; \quad K_{V T_{A B}}=0 \\
& K_{T V_{A B}}=0 ; \quad K_{T T_{A B}}=-\int_{\Gamma_{q}} N_{A}\left(h+4 \epsilon \sigma T^{3}\right) N_{B} \mathrm{~d} \Gamma
\end{aligned}
$$

Only the consistent tangent submatrix corresponding to the direct thermal field is non-zero, since the interface element is uncoupled and linear for the electric degree of freedom. Therefore, the tangent matrix is non-symmetric.

\section{Finite Element model}

Using the two special finite elements described in the previous Section, a CP1.4-127-045 TEC manufactured by Melcor [13] will be simulated. This TEC was chosen as representative of practical applications and is composed of 127 TC electrically connected in series, as in Figure 6. One of them was carefully measured to give the dimensions of Figure 7, where the upper alumina is in contact with the cold face and the lower with the hot. The maximum intensity of the TEC is 8.7 [A] (above that

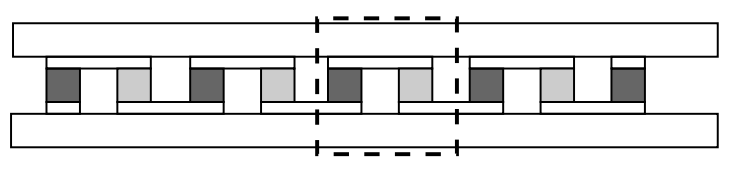

Domain modeling

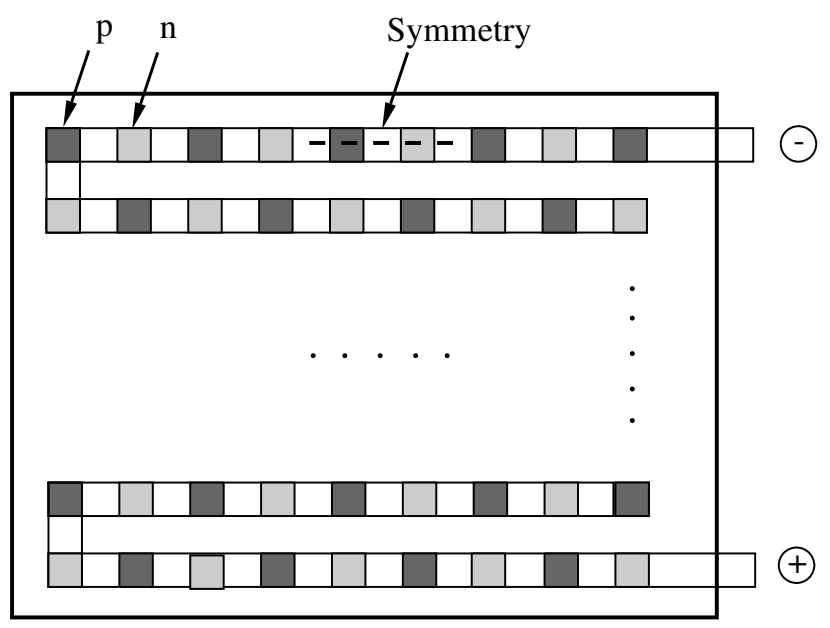

Figure 6: Representation of the CP1.4-127-045 thermoelectric cell, periodicity and symmetry. 
the irreversible Joule is prevalent) that under $T_{h}=T_{c}=50^{\circ} \mathrm{C}$ can extract a heat power $Q_{c t e c}=82.01$ [W] with voltage drop $V_{t e c}=15.33[\mathrm{~V}]$, according to the manufacturer, see Table 3.

Only half of the TC needs to be studied if it is assumed that the $T_{c}$ and $T_{h}$ distributions are constant, that is a reasonable hypothesis for medium and small devices. This is indicated by the symmetry line in the left view of Figure 7. If $T$ varies significantly, a mesh including more TC can be studied although at a higher computational cost. In any case the variation will depend on the media the TEC refrigerates, not on the TC itself. Assuming the conservation of charge hypothesis $\left(I_{t e c}\right.$ is he same everywhere in the TEC), only one TC needs to be studied, fact which is represented by periodicity lines in Figure 7.

On a surface of symmetry, the Neumann boundary conditions for electric and heat fluxes will be automatically set to zero $j_{c}=$ $q_{c}=0$, while on the two periodicity copper "cuts" (connections in the following), the prescribed flux $j_{c} \equiv j_{c f e}=I_{f e} / A_{c o n}$ is directly applied within the 2-D element (see Figure 8 and next Section) to the connection area $A_{c o n}$. Given that air and alumina are good electric isolators, no other electric flux condition is necessary.

Periodicity "cuts" are also present in the alumina lateral faces, represented in Figure 7 by sawed lines.

An alternative to the use of the 2-D element for the prescription of intensity is the term $I_{\Omega} \equiv I_{t e c} / \Omega_{e}\left(\Omega_{e}\right.$ would be the chosen element volume) from (7) in the adjacent 3-D elements, but with this solution some flux concentrations locally appear.

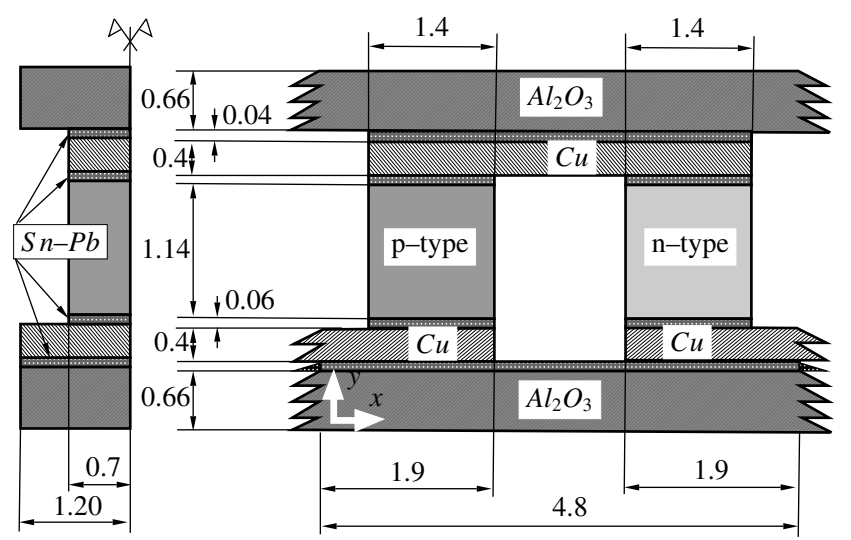

Figure 7: Dimensions [mm] of a CP1.4-127-045 thermocouple. Symmetry represented by flags, periodicity by sawed lines.

The 2-D element is also used in the cold and hot faces to force convection and radiation with air. The influence of other contact fluids, such as water, could be easily simulating by changing the parameter $h$.

The Dirichlet boundary conditions are applied at a surface of voltage reference $V=0$, chosen to be in the middle of the upper copper to preserve symmetry of the electric results, and in the cold face and hot face surfaces to the corresponding $T_{c}$ and $T_{h}$ (Figure 8). Notice that in spite of prescribing both Neumann and Dirichlet conditions in these surfaces, the problem is not of a mixed type, the 2-D element will only modify the related external unknown fluxes.

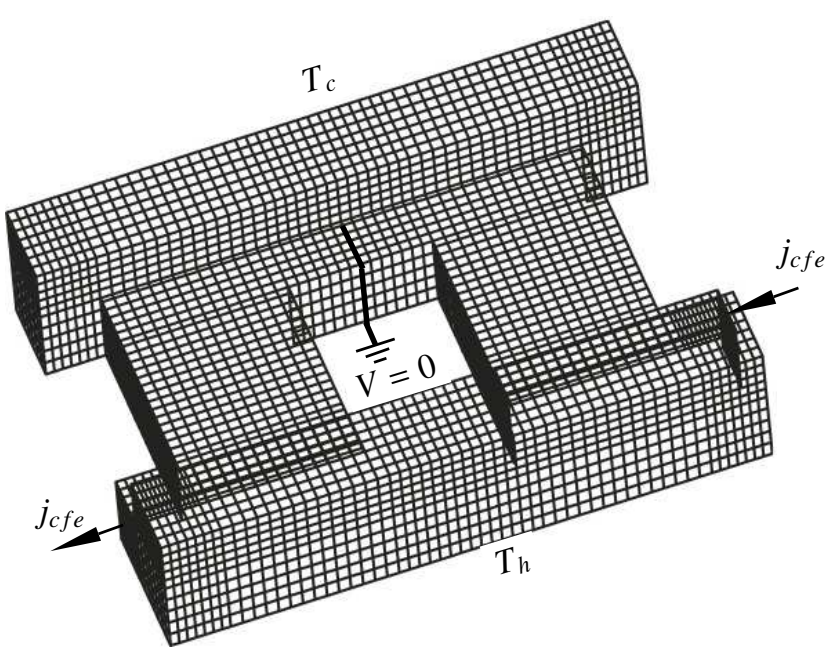

Figure 8: Mesh of 12,670 elements and prescribed boundary conditions.

\begin{tabular}{|c|c|c|c|c|}
\hline Iteration & 1 & 2 & 3 & 4 \\
\hline \hline$\|\mathbf{R}\|$ & 1.0002 & 0.0631 & 0.0029 & 0.00001 \\
\hline
\end{tabular}

Table 2: Quadratic residual norm convergence.

From the prescribed $T_{h}, T_{c}$ and $I_{f e}$, at any point of the domain the unknown volumetric variables $T, V, \mathbf{j}, \mathbf{q}$ as a function of space are found. From the difference between the $V$ values at each of the connections, the potential drop $V_{f e}$ (numerical counterpart of $V_{a f}$ in Figure 2) in a single TC can be easily computed. Similarly, from the addition of "reactions" (using the mechanical analogy) in the upper surface or cold face, the numerical total heat extracted $Q_{c f e}$ is estimated.

An optimal mesh size is now found using the parametrization of all dimensions from Figure 7. In Figure 9, the voltage drop between connexions $V_{f e}$ and the extracted heat $Q_{c f e}$ are plotted versus the total number of finite elements. The variables $V_{f e}$ and $Q_{c f e}$ have been normalized to their maximum values (coarse mesh) to show both convergences. With the restriction of conformity and similarity of finite element sizes, the resulting mesh is composed of 12,670 elements showed in Figure 8. It is interesting to note that $V_{f e}$ reaches the correct value with very few elements (about 320) but the power $Q_{c f e}$, proportional to a first derivative, needs a substantial higher number of at least 10,000 .

In order to obtain accurate results (the problem is highly nonlinear), the residual norm $\|\mathbf{R}\|$ must exhibit a quadratic convergence, see [18]. The order of convergence is obtained by $\ln \left\|\mathbf{R}_{k+1}\right\|=\ln \mu+m \ln \left\|\mathbf{R}_{k}\right\|$, where $k$ and $k+1$ are two successive iterations, $\mu$ is a parameter (about half of the CPU precision) and $m$ is the ratio of convergence. Table 2 shows the residual norms for each iteration; the ratio of convergence is $m \approx 2$, therefore the quadratic convergence is attained in only four iterations. 


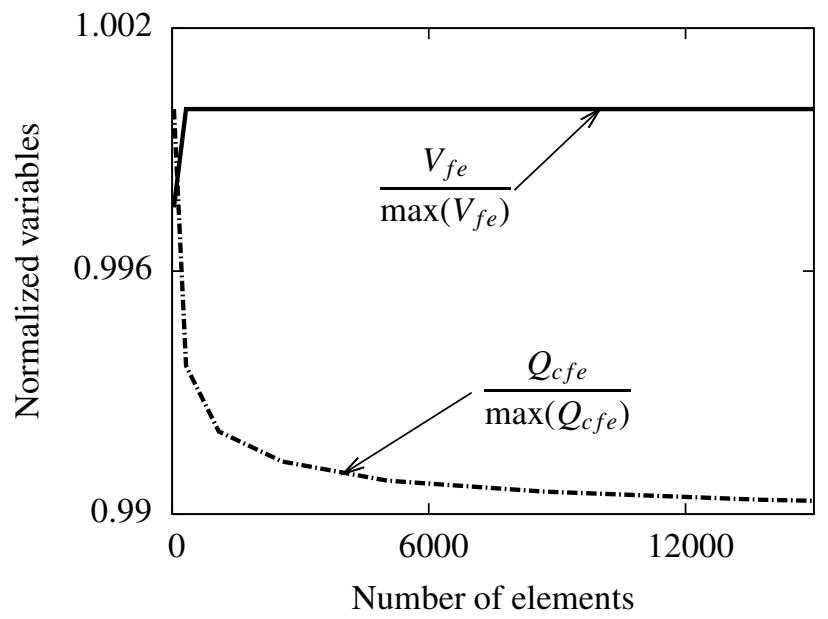

Figure 9: Voltage and thermal and flux convergence vs. number of elements.

\section{Thermoelectric Cell Cooler Simulation}

In this section, the FE and the mesh from Figure 8 have been used to simulate the TC. The temperature of the hot face has been maintained to $T_{h}=50^{\circ} \mathrm{C}$ in all cases, for intensities $I_{t e c}=$ $1.5,5.2,8.7$ [A]. Taking into account that only half of the TC is discretized, the prescribed intensity of the finite element model $I_{f e}$ will also be half of these values. The parameters calculated, directly or indirectly from the finite element results are:

- Voltage drop in the TC, taken directly from the difference of nodal values at both connectors, $V_{f e}$

- Voltage drop in the TEC, $V_{t e c}=127 V_{f e}$

- Maximum and minimum $T_{\max }$ and $T_{\min }$ temperatures in the TC, also from nodal values

- Heat taken from the cold face of a TC, $Q_{c f e}$

- Heat taken from the cold face $Q_{c t e c}=2 \cdot 127 Q_{c f e}$ by the TEC

- Coefficient of performance, $\mathrm{COP}=Q_{\text {ctec }} /\left(V_{\text {tec }} I_{\text {tec }}\right)$

\subsection{Finite Element distributions}

The finite elements formulated in Section 4 are used to simulate the TC described in Section 5, for $I_{t e c}=8.7$ [A] and $T_{h}=T_{c}=50^{\circ} \mathrm{C}$. These boundary conditions are chosen to maximize the studied effects, behaving the TEC as a heat pump that takes heat from the cold face (in fact a source) and gives it to the hot face (a sink).

Figure 10 top left shows the voltage distribution that decreases more or less linearly in the TE, while is constant in the other materials, good conductors or isolators. Inside the copper and closer to the cold face, an antisymmetric horizontal distribution is observed, due to the prescription of the reference zero potential. The total voltage drop is 0.1318 [V], that for the 127 TEC gives 16.74 [V] (see Table 3). This number is to be

\begin{tabular}{|c|c|c|c|}
\hline & Analytical & FEM & Melcor \\
\hline \hline$Q_{\text {ctec }}[\mathrm{W}]$ & 91.94 & 85.57 & 82.01 \\
\hline$V_{\text {tec }}[\mathrm{V}]$ & 14.71 & 16.74 & 15.33 \\
\hline$T_{\max }{ }^{\circ} \mathrm{C}$ & - & 78.5 & - \\
\hline$I_{\max }[\mathrm{A}]$ & 7.99 & 8.7 & 8.7 \\
\hline COP $[-]$ & 0.78 & 0.59 & 0.61 \\
\hline
\end{tabular}

Table 3: Thermoelectric cell performace for $T_{h}=T_{c}=50^{\circ} \mathrm{C}, I_{t e c}=8.7$ [A].

compared with 15.33 [V] given in the catalog, with a $9 \%$ difference. The drop occurs only in the TE, in the copper and even in the solders is very small due to their relatively high (two orders of magnitude for the first) electric conductivity. The analytical numbers in the table are computed using the simplified (3) and (4).

In the top right figure, the temperature distribution shows a parabolic distribution inside the TE, due to Joule. This is a very important fact, since the maximum temperature in the center is $78.5^{\circ} \mathrm{C}$, a $57 \%$ higher than the nominal $T_{h}$. Obviously, this increment substantially affects the heat from (3), see next paragraph. The alumina and copper close to the cold face are at a temperature similar to $T_{h}$; the value of $47.9^{\circ} \mathrm{C}$ in the surface, different from the prescribed $T_{h}$, is due to the discrete palette of colors used in the FE interpolation. In any case a slight gradient appears, close to the hot face to facilitate the transfer of heat power to it.

These distributions strongly depend on the boundary conditions. In Figure 11, $I_{t e c}$ and $T_{h}$ are maintained but $T_{c}$ varies through the functioning limits of the catalog. The distributions of $V$ (top) and $T$ (bottom) are drawn along a $y$ vertical line at the center of the p-type TE. As mentioned, voltage is linear or constant in all materials except in the TE were it varies linearly for $T_{c}=50^{\circ} \mathrm{C}$ and slightly non-linearly for the other values. It also varies in the solders, although it can not be appreciated due to the scale. The voltage drop decreases with the increase of $T_{c}$, since Seebeck is directly proportional to the temperature difference and, therefore, the conversion of thermal energy into electricity is reduced with this difference.

About the temperature (bottom figure), the variation of $T_{c}$ forces the final value in the left part of the plot. Joule is very clear inside the TE, specially for the heat pump mode, $T_{c}=50$, and $T$ is non-linear for all choices of $T_{c}$. Inside the copper and alumina Joule is not present, in the first due to its high conductivity $\gamma$ and in the second to the lack of electric current (very low $\gamma$ ), therefore $T$ is almost linear or even constant due to the lack of internal heat sources. At the copper-TE interface close to the hot face, $T$ almost coincides in all cases. Again the variation of the distribution in the $S n-P b$ solders is too small to be appreciated within the scale of the figures.

In the left column, middle and bottom Figure 10, the horizontal and vertical electric fluxes are shown. Since the electric intensity is constant, the maximum flux occurs in both coppers, where the conductive area is smaller, and zero in the alumina (an electric isolator). The flux is unidirectional (both horizontal and vertical in the copper and vertical but with different sign in the TE) except around some corners where a significant change 
of direction is represented by the concentration in both materials. In the TE's this flux is constant, in the copper the area by the TE is mostly vertical while in the rest mostly horizontal, with an abrupt change in the corners. This implies that the typical "rectangular" shape of the copper components is not optimal in the sense of electric conduction. Smaller fluxes than that of copper are present in the solders of copper and TE, due to their lower $\gamma$. Note that the application of the prescribed current $I_{t e c}$ with the special 2D finite element at the end of the external cut copper section ("connectors") does not produce any concentration.

The heat flux is shown in the second column, middle and bottom figures. The most interesting is the latter, vertical against $y$ (from the cold to the hot face), the direction along which the heat pumping occurs. In the TE, the flux is more intense closer to the hot face, due to the electric energy that is transformed into thermal and is directed against the direction of thermal conduction (towards the hot face). In the middle figure again a strong concentration in the internal corners of the copperalumina connection is appreciated, that will be quantified in the next paragraph. These concentrations are due to the sudden increment of the copper area, that forces the field lines to change direction towards the whole of the hot face and are antisymmetric with respect to the $x$ direction. The value $Q_{c t e c}=85.57$ [W] in Table 3 is taken from the finite element reactions of the alumina external surface in the cold face.

In spite of the 3D nature of the analysis, Figure 10 is represented in the $x-y$ plane, since the distributions are mostly $2 \mathrm{D}$. But there are some exceptions, in Figure 12 the 3D view of the vertical flux shows that the mentioned concentration corner also happens in the perpendicular plane. Also, in the hot face the flux is higher in the area vertical to the TE, which means that even if $T_{h}$ is forced to be constant the flux will be variable. This lack of uniformity also happens in the cold face, but is not visible due to the scale.

\subsection{Validation results}

The finite elements formulated in Section 4 are used to simulate the TC described in Section 5 with the mesh depicted in Figure 8 for three values of $I_{t e c}$, the maximum and minimum and another intermediate, and for $T_{h}=50^{\circ} \mathrm{C}$, all as function of $T_{c}$. These values are chosen to maximize the Peltier and Joule and to cancel Fourier. Thomson is directly included in the FE formulation with all its non-linearities and terms, although not in (3).

In Figure 13 the distributions for $Q_{c t e c}$ (top) and $V_{\text {tec }}$ (bottom) are shown. The correlation between the results, both in value and slope, given by the Melcor catalog and those of the current FE are very close for the extracted heat, even for the maximum intensity $I_{t e c}=8.7[\mathrm{~A}]$, that maximizes the irreversible Joule. For the voltage drop $V_{t e c}$, bottom figure, the agreement is perfect and good for the first two intensities and differs an almost constant $8.4 \%$ for the highest. This is due to the dependency of $\gamma$ with the temperature: since for this material decreases, Figure 4, when $T$ increases as in the TE center, $\gamma$ decreases with the result of a larger Joule and also a larger voltage drop needed. Obviously, this detrimental situation is worse for the maximum electric intensity and almost negligible for the low one. The situation is also visible in the extracted heat $Q_{c t e c}$ although less intense.

In any case this result is very sensitive to material properties and boundary conditions: for the high $I_{t e c}$, standard deviations bars (see subsection 7.3) show that with a small variation results almost coincide in the lower bound with those from the manufacturer. The simplified equation (3) gives values of $Q_{\text {ctec }}$ and specially $V_{\text {tec }}$ (plotted only for the maximum intensity) not that similar to those of the manufacturer. For the latter, the slope is different probably due to the absence of Thomson, that is based on the temperature dependency of $\alpha$.

\section{Sensitivity Analysis}

The aim of this section is to study the influence of the design variables on the COP. For this purpose, a Sensitivity Analysis (SA) is performed by combining the Monte Carlo (MC) and the FE analyses. The SA output permits to identify and quantify the main variables affecting the COP, with the intention to improve this performance variable.

\subsection{Monte Carlo Analysis}

Consider a physical model mathematically represented by:

$$
\phi_{i}=M\left(\xi_{j}\right)
$$

where $\phi_{i}$ are the $i$ dependent variables or responses, $\xi_{j}$ are the $j$ independent or design variables and $M$ is an operator that describes the model, the FE in the current work. The responses can be considered deterministic or probabilistic depending on the nature of the design variables.

The MC permits to obtain probabilistic responses by performing multiple evaluations of the model, using a sample of design variables previously generated. Then, this response is used to develop Uncertainty Analysis (UA) and SA. On one hand, UA calculates the uncertainties in the responses when the uncertainties in the design variables are known; thus, probability and cumulative distribution functions are obtained. Furthermore, two scalar parameters (mean $\mu$ and standard deviation $\sigma$ ) are calculated. On the other, SA determines the relationships between the uncertainties in the design variables and in the responses. There exist many available procedures to develop a $\mathrm{SA}$; in the present work, the multiple linear regression is used, see [14]. With this technique, the regression coefficients $\theta_{j}^{i}$ are obtained by minimizing the error between calculated and fitted parameters. In turn, $\theta_{j}^{i}$ are employed to define Standardized Regression Coefficients (SRC) $\Theta_{j}^{i}$, the scalar parameters that quantify the SA.

$$
\Theta_{j}^{i}=\theta_{j}^{i} \frac{\sigma_{\xi_{j}}}{\sigma_{\phi_{i}}}
$$

where $\sigma_{\phi_{i}}, \sigma_{\xi_{j}}$ are the standard deviations of the responses and of the design variables, respectively. 


\begin{tabular}{|c|c|c|}
\hline Property & $\sigma$ & Notation \\
\hline \hline $\mathrm{Al}_{2} \mathrm{O}_{3}$ thick. & $5 \%$ & $\Theta_{1}$ \\
\hline$S n$ - $\mathrm{P} b$ thick. (lower) & $5 \%$ & $\Theta_{2}$ \\
\hline $\mathrm{Cu}$ length & $5 \%$ & $\Theta_{3}$ \\
\hline TE length & $5 \%$ & $\Theta_{4}$ \\
\hline$S n-P b$ thick. (upper) & $5 \%$ & $\Theta_{5}$ \\
\hline$T_{h}$ & $1 \%$ & $\Theta_{6}$ \\
\hline$T_{c}$ & $1 \%$ & $\Theta_{7}$ \\
\hline$I_{t e c}$ & $1 \%$ & $\Theta_{8}$ \\
\hline$\kappa\left(A l_{2} \mathrm{O}_{3}\right)$ & $5 \%$ & $\Theta_{9}$ \\
\hline$\alpha_{0}, \alpha_{1}, \alpha_{2}(\mathrm{TE})$ & $5 \%$ & $\Theta_{10,11,12}$ \\
\hline$\gamma_{0}, \gamma_{1}, \gamma_{2}(\mathrm{TE})$ & $5 \%$ & $\Theta_{13,14,15}$ \\
\hline$\kappa_{0}, \kappa_{1}, \kappa_{2}(\mathrm{TE})$ & $5 \%$ & $\Theta_{16,17,18}$ \\
\hline$\gamma(\mathrm{Cu})$ & $5 \%$ & $\Theta_{19}$ \\
\hline$\kappa(\mathrm{Cu})$ & $5 \%$ & $\Theta_{20}$ \\
\hline$\gamma(S n-P b)$ & $5 \%$ & $\Theta_{21}$ \\
\hline$\kappa(S n-P b)$ & $5 \%$ & $\Theta_{22}$ \\
\hline
\end{tabular}

Table 4: Standard deviations and SRC notation for the design variables. Upper solder between $\mathrm{Cu}$ and $\mathrm{Al}_{2} \mathrm{O}_{3}$, lower between $\mathrm{Cu}$ and $\mathrm{Bi}_{2} \mathrm{Te}_{3}$.

\subsection{Problem definition}

The main task in developing a SA is the choice of the design variables and their distribution functions, often from experimental considerations, see [19]. In the present work, the design variables are: a) the geometric dimensions, b) the material properties of all the materials and c) the prescribed $I_{t e c}, T_{c}$, $T_{h}$. Their distribution functions are not reported by the manufacturer and they are assumed to be normally distributed. The mean of the design variables are also assumed to be those in Table 1 and Figure 7, while the SRC notation and the standard deviation are given in Table 4. These are assumed to be: i) $5 \%$ for the geometric parameters, ii) $1 \%$ for the prescribed variables, mostly due to variations in the temperature distributions and errors in the electric source, and finally iii) $5 \%$ for the material parameters measurement error, see [20]. In Table 4 the TM properties $\alpha, \gamma$ and $\kappa$ are represented by three variables, corresponding to the three polynomial coefficients in (6).

In order to generate the sample of the random variables, the Latin hypercube technique is used since the convergence is faster than using the random technique, see [14]. Finally, an optimized sample of size $m=100$ to reduce the CPU cost and guarantee the convergence was calculated by the procedure also in [14]. To sum up, there are 22 design variables normally distributed, three responses $\left(V_{t e c}, Q_{c t e c}, \mathrm{COP}\right)$ and the model $M$ is solved by the FE described in Section 4.

\subsection{SA Results}

The UA results obtained are shown in Figure 14, where the probability distribution functions of the responses for the case studied in subsection 6.1 are presented. The means agree well with the deterministic results showed in Figures 13 and Table 3, implying that the UA results are accurate. The type of distribution functions are obtained using the Jarque-Bera test; concluding that the responses $V_{t e c}, Q_{c t e c}$ are not normally distributed while the COP is. This distribution types are expected since

\begin{tabular}{|c|c|c|c|c|}
\hline & & $Q_{\text {ctec }}[\mathrm{W}]$ & $V_{\text {tec }}[\mathrm{V}]$ & COP [-] \\
\hline \hline & Melcor & 82.01 & 15.33 & 0.61 \\
\hline i) & $\alpha\left(T_{m}\right), \kappa\left(T_{m}\right), \gamma\left(T_{m}\right)$ & 83.073 & 15.798 & 0.604 \\
\hline ii) & $\alpha(T), \kappa(T), \gamma(T)$ & 85.577 & 16.738 & 0.587 \\
\hline iii) & $\alpha(T), \kappa(T), \gamma\left(T_{m}\right)$ & 88.742 & 15.824 & 0.644 \\
\hline iv) & $\alpha\left(T_{m}\right), \kappa(T), \gamma(T)$ & 79.362 & 16.738 & 0.545 \\
\hline
\end{tabular}

Table 5: Thermoelectric cell performance for $T_{h}=T_{c}=50^{\circ} \mathrm{C}, I_{t e c}=8.7$ [A].

both voltage and extracted heat are non-linear (due to Joule and material nonlinearities) while the COP is a normalized variable.

In Figure 13 (bottom and $I_{\text {tec }}=8.7$ [A]) the voltage value was shown with error-bars. The mean (large circle) calculated here agrees well with the deterministic results (small circle) while the lower end of the standard deviations (straight bars) slightly overlap the manufacturer curves. Therefore, the probabilistic model with reasonable values of standard deviations agrees with the manufacturer results.

The SRC's obtained from the SA are shown in Figures 15. The top one shows that the $V_{\text {tec }}$ is most sensitive to the design variables $\Theta_{4}$ (TE length) and $\Theta_{13}, \Theta_{14}, \Theta_{15}$, the $\gamma$ coefficients. This is predictable, since Joule is a bulk effect that depends on the TE 's length and since the voltage drop is proportional to the resistivity (inverse of $\gamma$ ). The least relevant coefficient of the electric conductivity is $\gamma_{2}$, since the dependence on temperature of $\gamma$ is practically linear in the studied range, see Figure 4.

The SRC's for $Q_{c t e c}$ are shown in Figure 15 middle. Now the most sensitive design variable is $\Theta_{10}\left(\alpha_{0}\right)$, since the Peltier heat strongly depends on Seebeck. Again, $\alpha_{2}$ is not relevant since the material property is fairly linear, Figure 4 , and $\Theta_{4}, \Theta_{13}, \Theta_{14}$, $\Theta_{15}$ are relevant, for the same reasons as those of $V_{t e c}$. Finally, since COP is a relation of the previous responses, its sensitivities are the same, as shown in the bottom figure. Among the rest, input current $\Theta_{8}$ is the only one slightly sensitive, the rest can be considered as numerical noise; in particular the sensitivity of $\kappa$ is not relevant since conduction is cancelled by the choice $T_{h}=T_{c}=50^{\circ} \mathrm{C}$.

\section{Discussion}

The design variables that need to be controlled to design a good thermoelectric cell are: TE length, electrical conductivity and Seebeck coefficients. These three results from Section 7 agree with the already known facts: a good TM needs a high $\alpha$ to maximize Peltier, also a high $\gamma$ to reduce Joule and, although not studied here, a low $\kappa$ to reduce heat transfer. The variability of $\kappa$ is not influential (see Figure 15), therefore its variation will not be considered.

The objective of this Section is to discuss the influence of the temperature dependence of the material properties in the TEC performance. For that, two computations are performed: the first for the extracted heat $Q_{c t e c}$ and the necessary voltage drop $V_{\text {tec }}$ with fixed $T_{c}=T_{h}=50^{\circ} \mathrm{C}, I_{\text {tec }}=8.7$ [A], and the second for the COP and again $Q_{c t e c}$ with $T_{c}=15, T_{h}=50^{\circ} \mathrm{C}$ and varying $I_{t e c}$. 
In both, the results are compared using on one hand all properties varying with $T$ from (6), and on the other some or all of them constant. Four hypotheses are contemplated:

i) constant $\alpha\left(T_{m}\right), \gamma\left(T_{m}\right), \kappa\left(T_{m}\right)$ as in [2]

ii) temperature-dependent $\alpha(T), \gamma(T), \kappa(T)$ as in Section 6.1

iii) idem for $\alpha(T), \kappa(T)$ and constant for $\gamma\left(T_{m}\right)$

iv) idem for $\gamma(T), \kappa(T)$ and constant for $\alpha\left(T_{m}\right)$

The constant properties are obtained using the average temperature between the external faces, $T_{m}=\left(T_{c}+T_{h}\right) / 2$. The results are shown in Table 5, where the performance is calculated with the FE for the four hypotheses. For comparison purposes, the results from the manufacturer are also included.

The COP increases from iv) to ii) by $7.7 \%$. This result approximately agrees with that reported in [21], where it was concluded that the consideration of Thomson increases the COP by $5-7 \%$. The COP decreases from iii) to ii) by $8.8 \%$. This is due to the decreasing temperature-dependence of $\gamma$, see Figure 4, that will be lower under $78^{\circ} \mathrm{C}$ in the TE middle than under $T_{m}$. Therefore, Joule is increased, forcing the potential drop to be larger and the COP lower. In Table 5 and as showed in Figure 15 , it can be appreciated that $V_{\text {tec }}$ is sensible to the temperature dependence of $\gamma$, but not to that of $\alpha$. Finally, $Q_{\text {ctec }}$ is sensible to the dependence of both $\alpha$ and $\gamma$.

In Figure 16, COP (vertical axis) and $Q_{c t e c}$ (horizontal) values are represented for increasing intensities and for the four hypotheses. Starting with a small $I_{t e c} \approx 1.8$ [A], both COP and $Q_{\text {ctec }}$ increase since Peltier is predominant over Joule. When $I_{\text {tec }} \approx 3.4$ [A], Peltier is still predominant, but the external electric power $V_{t e c} I_{t e c}$ (denominator in (7)) is higher. The consequence is that $Q_{\text {ctec }}$ keeps increasing but COP decreases. However, at $I_{t e c} \approx 8.7$ [A], (the maximum recommended by the manufacturer) the value of Joule becomes larger than that of Peltier, and both variables decrease.

Consider now a constant $\mathrm{COP} \approx 0.3$, then two intensities are possible: one with a low extracted heat but also low electric power used, and another with a high heat but also high electric power.

For $I_{t e c} \leq 3.4$ and for $I_{t e c} \geq 17.8$ [A] the differences between the four mentioned hypotheses are very small since Peltier and Joule are very dominant, respectively. This result agrees whit that reported in [21]. Note that the FE cannot produce results for intensities larger than 17.8 [A] due to numerical overflows.

Between 3.4 and 17.8 [A], there are relevant differences among the hypotheses: for iv), $Q_{\text {ctec }}$ has the lowest value due to Thomson. For iii), $Q_{c t e c}$ is highest, since the potential drop is also highest due to the increase in Joule.

Summarizing, the most important conclusion obtained in the present work is that the decreasing temperature-dependence of $\gamma$ is more relevant for the COP than that of $\alpha$.

\section{Acknowledgments}

This research was partially supported by the Ministry of Education through Grant FPU AP-2006-02372 and also from grants
MICINN BIA-2008-00522, MCyT DPI 2002-04472-C02-02 and Excelencia Junta Andalucía P08-TEP-3641.

\section{References}

[1] S. Riffat, X. Ma, Thermoelectrics: a review of present and potential applications, Applied Thermal Engineering 23 (2003) 913-935.

[2] D. Rowe (Ed.), CRC Handbook of Thermoelectrics, CRC Press, 1995.

[3] J. Chen, Z. Yan, L. Wu, Nonequilibrium thermodynamic analysis of a thermoelectric device, Energy 22 (10) (1997) 979-985.

[4] M. Huang, R. Yen, A. Wang, The influence of the Thomson effect on the performance of a thermoelectric cooler, International Journal of Heat and Mass Transfer 48 (2005) 413-318.

[5] O. Yamashita, Effect of linear an non-linear components in the temperature dependences of thermoelectric properties on the cooling performance, Applied Energy 86 (2009) 1746-1756.

[6] D. Gavela, J. Pérez-Aparicio, Peltier pellet analysis with a coupled, nonlinear 3D finite element model, in: 4th European Workshop on Thermoelectrics, 1998.

[7] J. Pérez-Aparicio, D. Gavela, 3D, non-linear coupled, finite element model of thermoelectricity, in: 4th European Workshop on Thermoelectrics, 1998.

[8] J. Pérez-Aparicio, R. Taylor, D. Gavela, Finite element analysis of nonlinear fully coupled thermoelectric materials, Computational Mechanics 40 (2007) 35-45.

[9] E. Antonova, D. Looman, Finite elements for thermoelectric device analysis in ANSYS, in: International Conference on Thermoelectrics, 2005.

[10] D. Ebling, M. Jaegle, M. Bartel, A. Jacquot, H. Bottner, Multiphysics simulation of thermoelectric systems for comparison with experimental device performance, Journal of Electronic Materials 38 (7) (2009) 14561461.

[11] C. Cheng, S. Huang, T. Cheng, A three-dimensional theoretical model for predicting transient thermal behavior of thermoelectric coolers, International Journal of Heat and Mass Transfer 53 (2010) 2001-2011.

[12] J. Oden, T. Belytschko, I. Babuska, T. Hughes, Research directions in computational mechanics, Comput. Methods Appl. Mech. Engrg. 192 (2003) 913-922.

[13] MELCOR, Thermoelectric Handbook, Melcor, a unit of Laird Technologies, http://www.lairdtech.com, 2000.

[14] R. Palma, G. Rus, R. Gallego, Probabilistic inverse problem and system uncertainties for damage detection in piezoelectrics, Mechanics of Materials 41 (2009) 1000-1016.

[15] A. Arenas, J. Vázquez, M. Sanz-Bobi, R. Palacios, Performance of a thermoelectric module using the thermodynamic relationship temperatureentropy (T-S), in: XIX International Conference of Thermoelectrics, 2000.

[16] H. Chua, K. Ng, X. Xuan, C. Yap, J. Gordon, Temperature-entropy formulation of thermoelectric thermodynamic cycles, Physical Review E 65 (2002) 056111.

[17] R. Taylor, FEAP A Finite Element Analysis Program: User Manual, University of California, Berkeley, http://www.ce.berkeley.edu/feap, 2010.

[18] O. Zienkiewicz, R. Taylor, J. Zhu, The Finite Element Method: The Basis, Elsevier Butterworth-Heinemann, 2005.

[19] A. Saltelli, K. Chan, E. Scott, Sensitivity Analysis, John Wiley and Sons, 2000.

[20] G. Smith, R. Wolfe, Thermoelectric properties of bismuth-antimony alloys, Journal of Applied Physics 33 (3).

[21] A. Chakraborty, B. Saha, S. Koyoma, K. Ng, Thermodynamic modelling of a solid state thermoelectric cooling device: Temperature-entropy analysis, International Journal of Heat and Mass Transfer 49 (2006) 35473554 . 

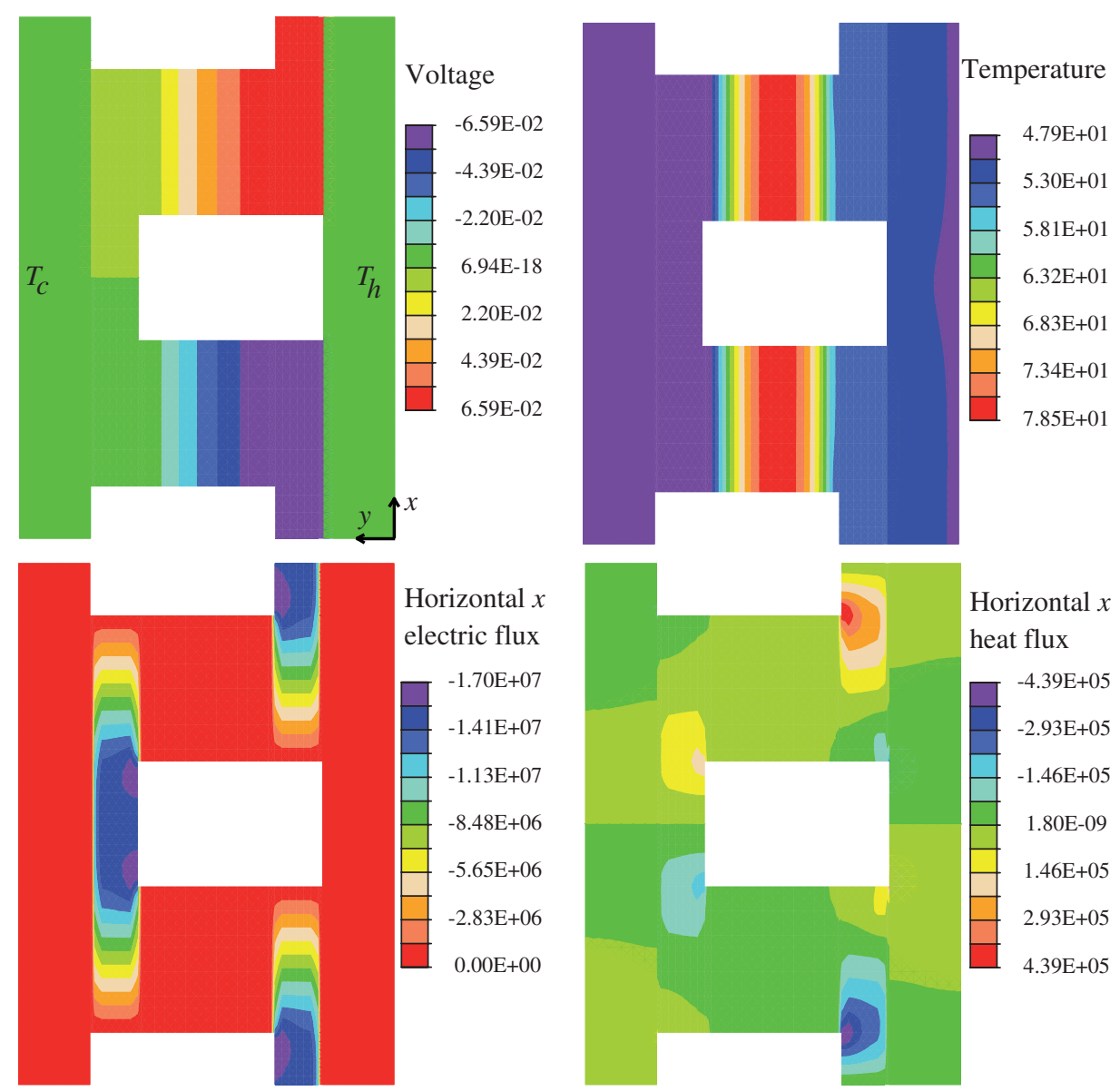

Horizontal $x$ heat flux
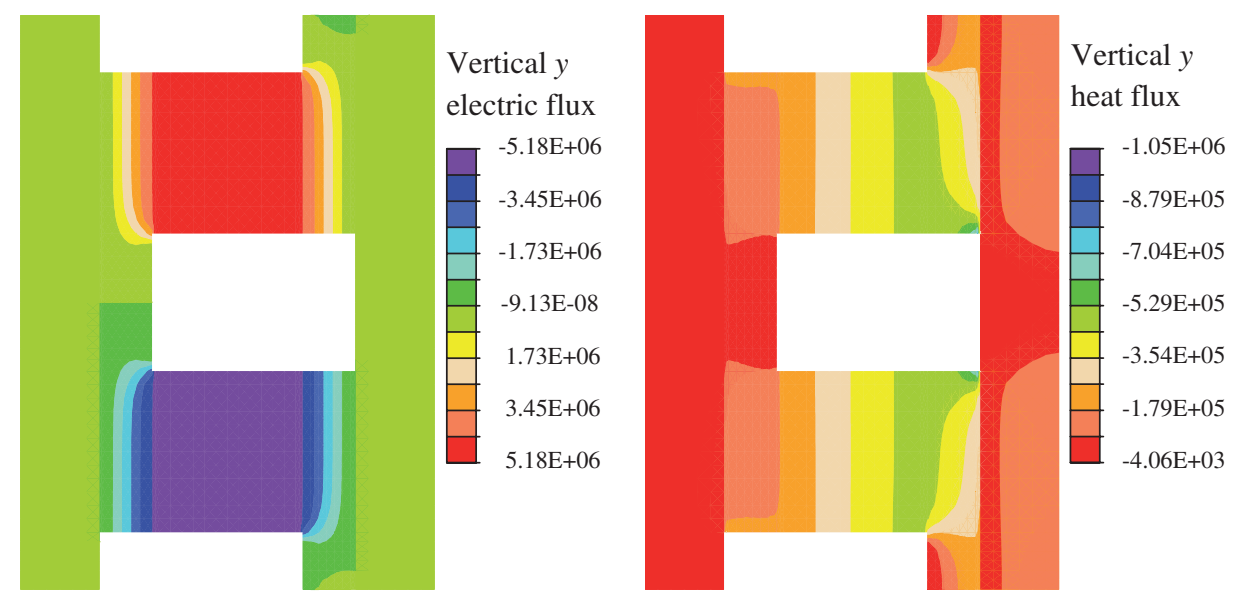

Figure 10: Top: Voltage (left) and Temperature (right). Middle: Horizontal electric (left) and thermal (right) fluxes. Bottom: Vertical electric (left) and thermal (right) fluxes, all for $I_{f e}=8,7[\mathrm{~A}], T_{c}=T_{h}=50^{\circ} \mathrm{C}$. 

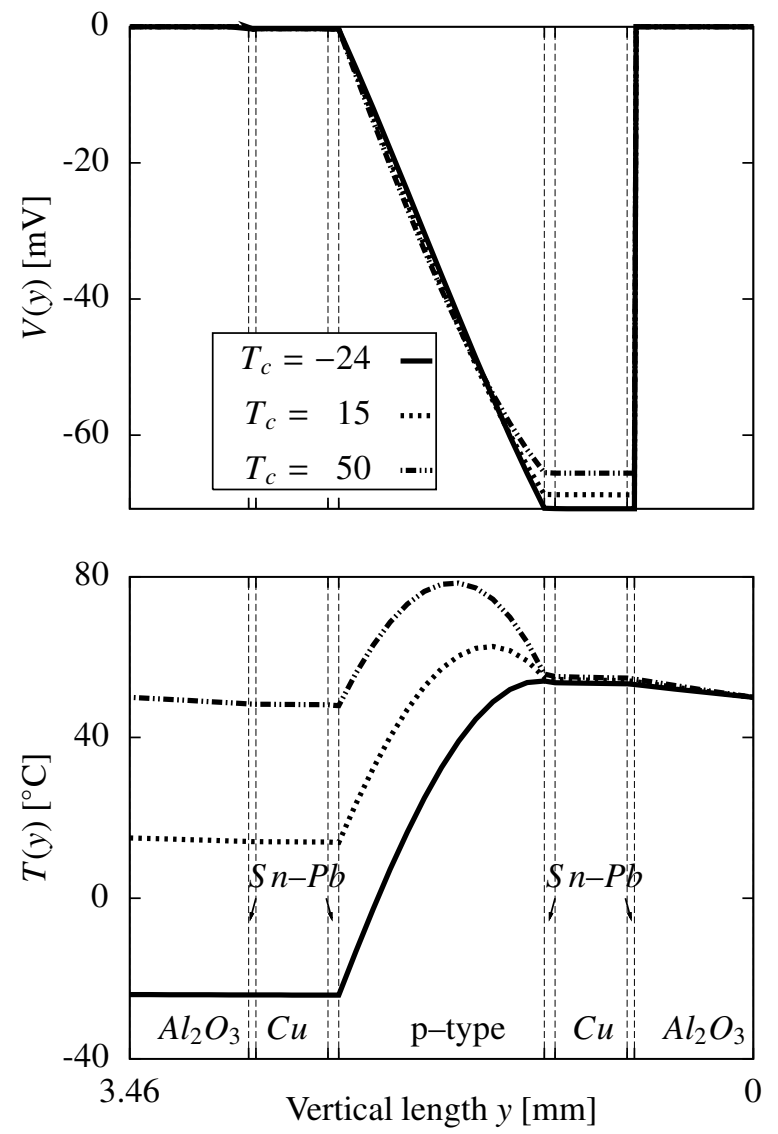

Figure 11: Vertical temperature and voltage distributions for $T_{h}=50^{\circ} \mathrm{C}$. Origin at the thermoelement bottom-left.
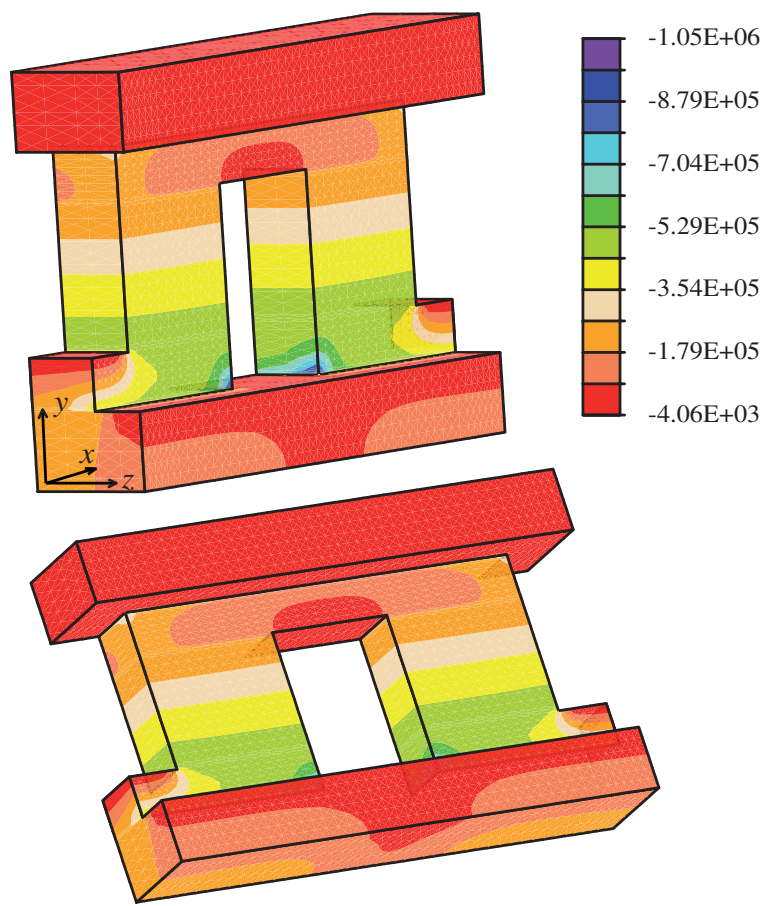

Figure 12: 3D view of the vertical heat flux $q_{y}$ for $I_{f e}=8,7[\mathrm{~A}], T_{c}=T_{h}=$ $50^{\circ} \mathrm{C}$.
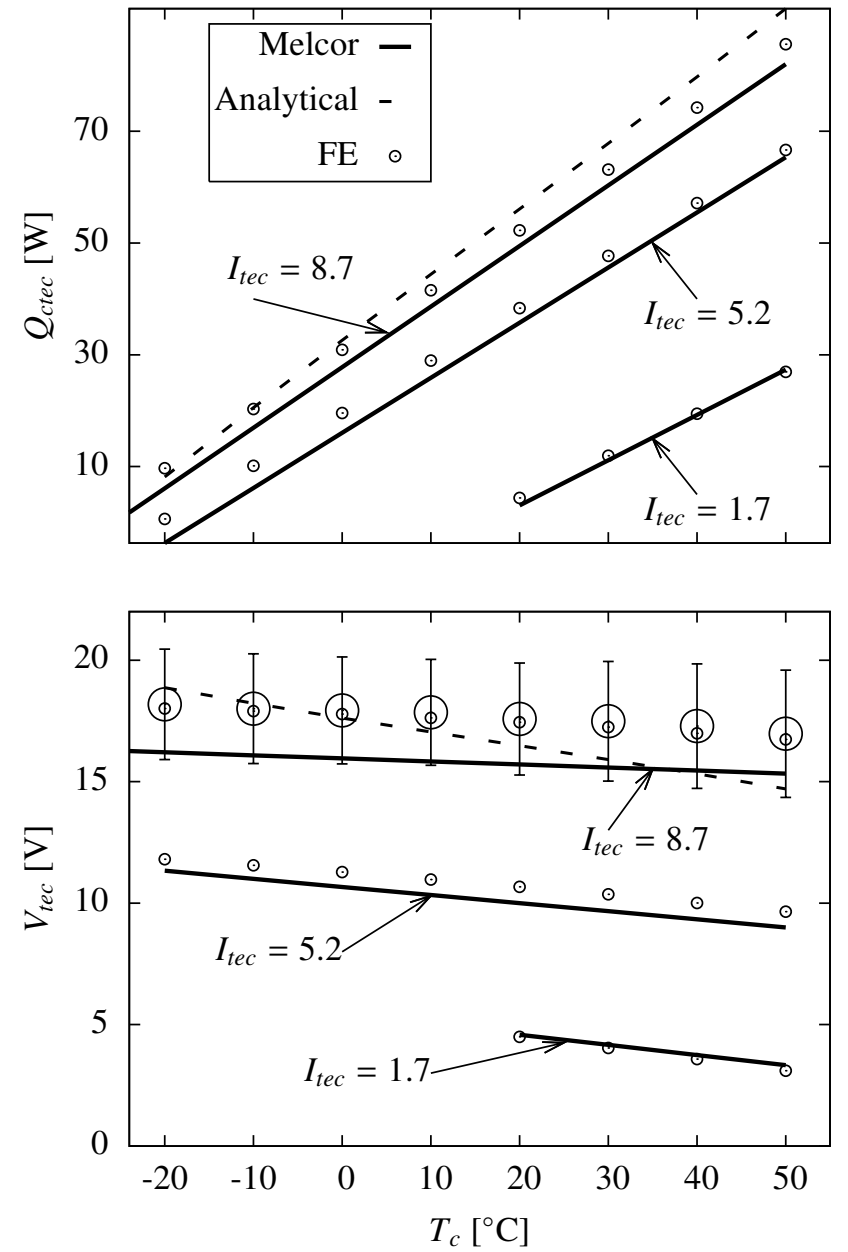

Figure 13: Comparison of analytical formulae (Rowe [2]), current (finite element) and manufacturer (Melcor, [13]) heat extracted and voltage drop for the functioning range $I_{t e c}, T_{c}$ and for $T_{h}=50^{\circ} \mathrm{C}$. 

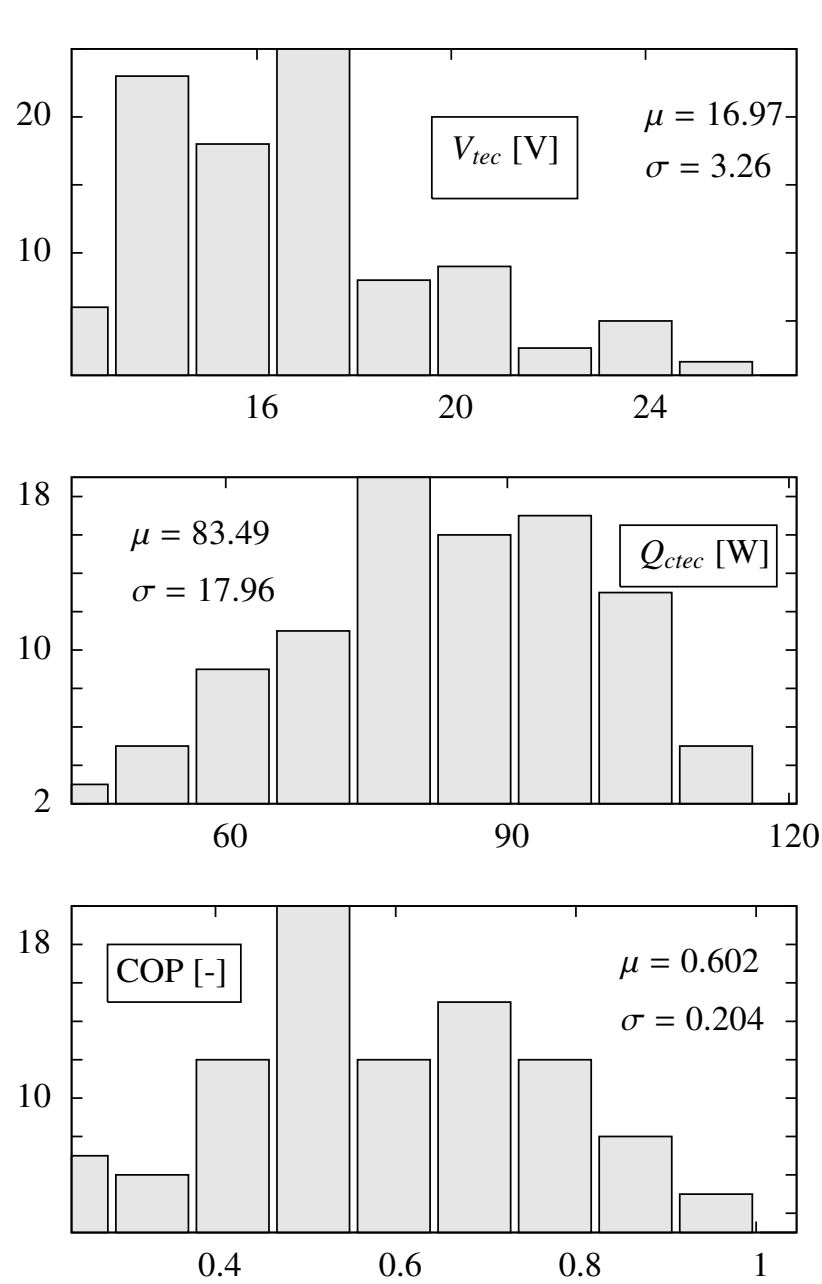

Figure 14: Probability distribution function, $T_{c}=T_{h}=50^{\circ} \mathrm{C}, I_{\text {tec }}=8.7$ [A].
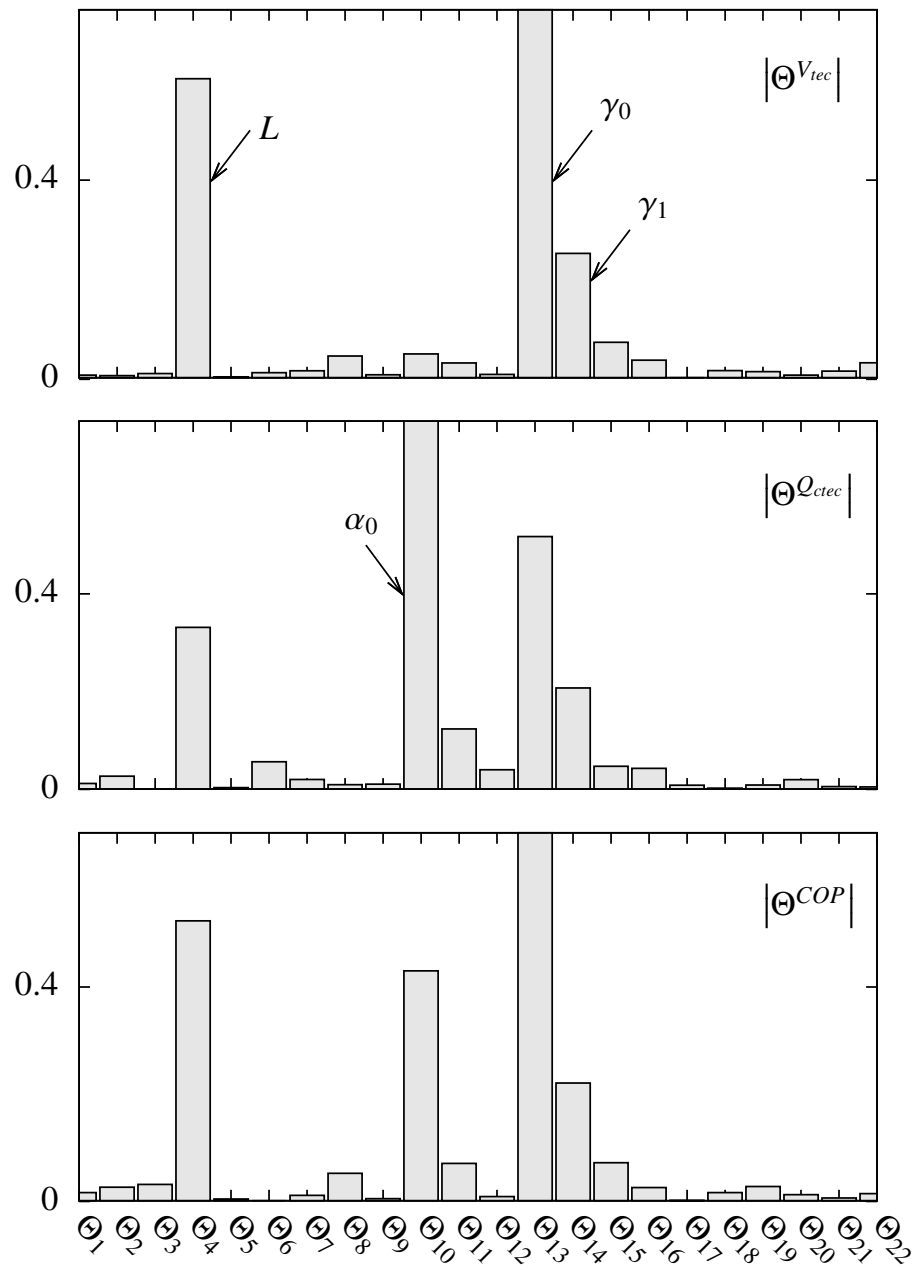

Figure 15: Standardized regression coefficient in absolute value for $T_{c}=T_{h}=$ $50^{\circ} \mathrm{C}$ and $I_{\text {tec }}=8.7[\mathrm{~A}]$. Notation in Table 4.

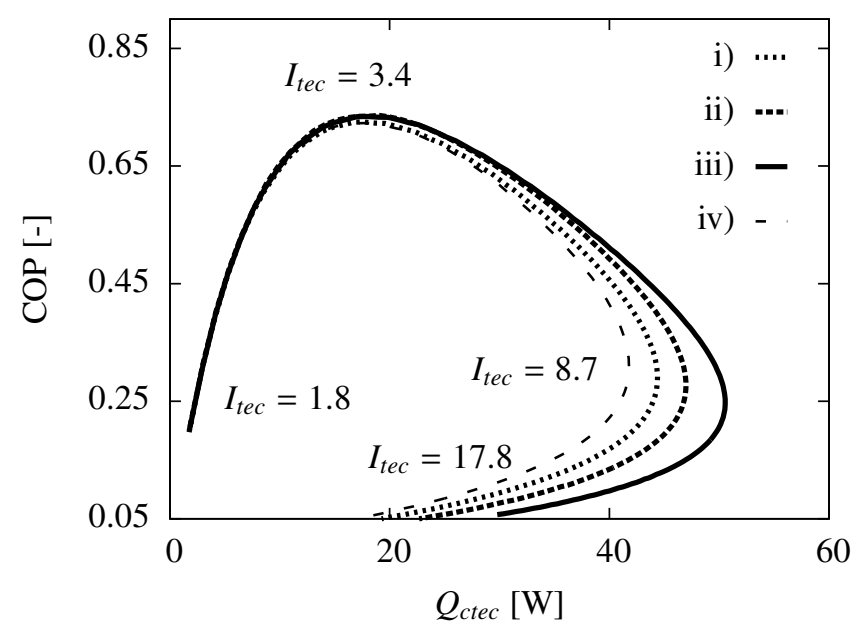

Figure 16: $\mathrm{COP}$ and $Q_{c t e c}$ for increasing electric intensity, $T_{h}=50^{\circ} \mathrm{C}, T_{c}=$ $15^{\circ} \mathrm{C}$. Thermal conductivity $\kappa$ temperature dependent. 